\title{
Erratum to: Cadaver Biopsies Positively Impact the Medical Student Educational Experience
}

\author{
Jonathan L. Lavezo ${ }^{1}$ - Elmus G. Beale ${ }^{2}$ - Naomi L. Lacy ${ }^{3}$ Tanis Hogg ${ }^{3}$ • \\ Osvaldo Padilla $^{3,4}$ • Niti Manglik ${ }^{3,4}$ • Veronica Y. Velasco ${ }^{3,4}$
}

Published online: 11 September 2017

(C) International Association of Medical Science Educators 2017

Erratum to: Med.Sci.Educ.

https://doi.org/10.1007/s40670-017-0440-2

Corrected figure 1 appears here.

The online version of the original article can be found at https://oi.org/ $10.1007 / \mathrm{s} 40670-017-0440-2$

Veronica Y. Velasco

veronica.velasco@ttuhsc.edu

1 Department of Pathology, Stanford University Hospital and Clinics, Stanford, California, USA

2 Faculty of Community Medicine, Oxley College of Health Sciences, The University of Tulsa, Tulsa, OK, USA

3 Department of Medical Education, Paul L. Foster School of Medicine, Texas Tech University Health Sciences Center-El Paso, 5001 El Paso Drive, El Paso, TX 79905, USA

4 Department of Pathology, Paul L. Foster School of Medicine, Texas Tech University Health Sciences Center-El Paso, El Paso, TX, USA 
1.) I feel that students should be allowed to continue to perform cadaver biopsies during anatomy lab.

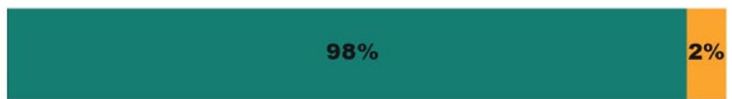

2.) I feel that this exercise was helpful in my preparation for the USMLE Step 1.

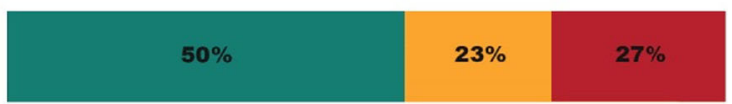

3.) The use of cadaver biopsies helped me formulate a complete understanding of my cadaver for Tank Side Grand Rounds.

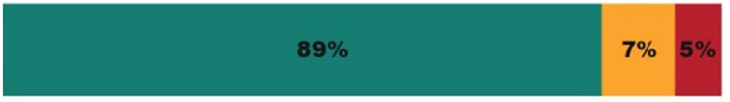

4.) The use of cadaver biopsies has increased my interest in choosing pathology as a residency specialty.

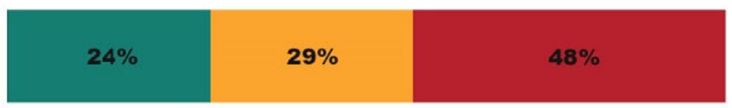

5.) The use of cadaver biopsies helped me understand clinical scenarios with respect to patient care during the 3rd year of medical school.

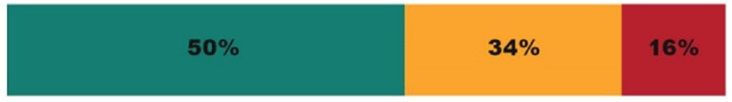

6.) The use of cadaver biopsies has increased my interest in pathology/lab results for patients during the 3rd year of medical school.

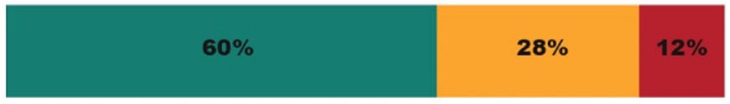

7.) The use of cadaver biopsies was helpful to learn clinical connections during the first two years.

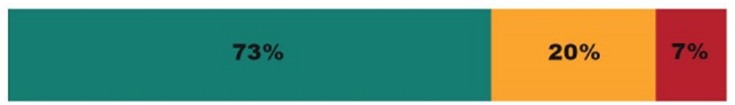

8.) The use of cadaver biopsies was helpful to make connections between different basic sciences during the first two years.

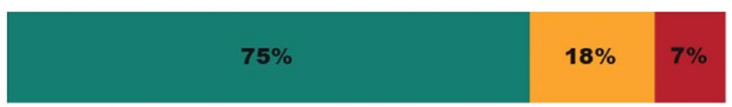

9.) The use of cadaver biopsies was helpful in improving my understanding of pathology during the first two years.

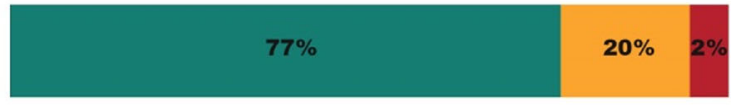

Percentage of 44 Total Response (n) Agree

Percentage of 44 Total Responses (n) Neutral

Percentage of $\mathbf{4 4}$ Total Responses (n) Disagree

Fig. 1 Responses to cadaver biopsy statements. Each horizontal bar shows the marginal response category frequencies for each of the cadaver biopsy statements for all 44 respondents to each of the 9 survey statements 